\title{
Aspectos epidemiológicos de malária autóctone na mata atlântica, litoral norte, Estado de São Paulo, 1985 - 2006
}

\author{
Epidemiological aspects of autochthonous malaria in the Atlantic forest \\ area of the northern coast of the State of São Paulo, 1985 - 2006
}

\author{
Gisela Rita Alvarenga Monteiro Marques', Maria Lúcia Fadel Condino', \\ Lígia Leandro Nunes Serpa ${ }^{1}$ e Thaís Valladão Mello Cursino ${ }^{1}$
}

\begin{abstract}
RESUMO
Com o objetivo analisar a distribuição espacial e temporal de casos de malária autóctone, segundo: densidade parasitária, sintomas e atividade por ocasião da infecção. Foi feito estudo descritivo retrospectivo de 1985 a 2006, nos municípios de Ubatuba, Caraguatatuba, São Sebastião e Ilhabela. Os dados foram obtidos na ficha de investigação epidemiológica da Superintendência de Controle de Endemias e Sistema de Informação de Notificação de Agravos à Saúde. Dos 83 casos, 77\% eram do sexo masculino. São Sebastião e Caraguatatuba apresentaram maior número de casos. Os indivíduos mais acometidos encontraram-se em idade produtiva com Plasmodium vivax, e densidade parasitária de um parasita a cada dois ou mais campos microscópicos. Lazer foi a atividade mais exercida pelos pacientes por ocasião da infecção. Os principais sintomas foram febre, cefaléia, sudorese e mialgia. Foram registrados 4 indivíduos assintomáticos. Esses dados devem contribuir para a melhoria das ações de descentralização do controle da malária na região considerando-a na suspeita clínica e/ou epidemiológica, laboratório de referência e recursos humanos treinados para o diagnóstico e tratamentos adequados.
\end{abstract}

Palavras-chaves: Anopheles. Controle. Vigilância epidemiológica. Malária.

\section{ABSTRACT}

This study had the aim of analyzing the spatial and temporal distribution of autochthonous malaria cases according to parasite density, symptoms and activity at the time of infection. A retrospective descriptive study was undertaken in the municipalities of Ubatuba, Caraguatatuba, São Sebastião and Ilhabela, covering 1985 to 2006. The data were obtained from the epidemiological investigation files of the Superintendency of Endemic Disease Control and the Information System for Health Threat Notification. Out of the 83 cases notified, 77\% were male. São Sebastião and Caraguatatuba presented the largest numbers of cases. The individuals most affected with Plasmodium vivax were at productive ages, with a parasite density of one parasite for every two or more microscope fields. At the time of becoming infected, these patients were mostly doing leisure activities. The main symptoms were fever, headache, sweating and myalgia. Four asymptomatic cases were detected. These data are expected to contribute towards improving the decentralized malaria control actions in the region, through considering malaria among the clinical and/or epidemiological suspicions, with a reference laboratory and trained personnel for adequate diagnosis and treatment.

Key-words: Anopheles. Control. Epidemiological surveillance. Malaria.

A malária é reconhecida como grave problema de saúde pública, sendo a doença parasitária mais importante da região tropical, com altas taxas de morbidade, mortalidade, $\mathrm{e}$ aproximadamente 1 milhão de mortes/ano no mundo. Caracterizase como doença infecciosa febril, aguda, causada por protozoários e transmitidas por insetos vetores ${ }^{812}$.

No Brasil, aproximadamente $99 \%$ dos casos concentram-se na Região Amazônica. A maioria ocorre em áreas rurais, mas há também registro da doença em áreas urbanas. Na Região Sudeste, a malária autóctone ocorre em todos os estados em áreas cobertas pela Mata Atlântica ${ }^{15}$.

Em 2006, foram notificados 57 casos autóctones de malária no Estado de São Paulo, que representaram 42\% do total de casos registrados na região extra-amazônica, correspondendo a um aumento de $90 \%$ em relação ao ano anterior ${ }^{9}$.

1. Seção Técnica de Pesquisa em Vetores, Superintendência de Controle de Endemias, Taubaté, SP.

Endereço para correspondência: Dr ${ }^{a}$ Gisela Rita Alvarenga Monteiro Marques. Seção Técnica de Pesquisa em Vetores/SUCEN São Paulo. Praça Coronel Vitoriano 23, Jardim Santa Clara, 12020-020 Taubaté, SP.

Tel: 5512 3632-7616

e-mail: giselammarques@uol.com.br

Recebido para publicação em 27/02/2008

Aceito em 17/07/2008 
Segundo Machado e cols ${ }^{7}$, a dinâmica de transmissão da malária é variável entre as regiões, considerando a interação de diferentes fatores de risco. Para tanto, aspectos de natureza ecológica, econômica, sociocultural e de saúde pública determinam diferenças na ocorrência da doença.

A transmissão natural, no Estado de São Paulo, ocorre em duas regiões geográficas distintas. A primeira, representada pela região oeste, em especial nas áreas de influência das bacias hidrográficas dos rios Paraná, Paranapanema e São José dos Dourados. A segunda, representada pela região da Serra do Mar, ecossistema ainda em boa parte preservado e com presença de Mata Atlântica, onde anofelinos do subgênero Kerteszia podem ser encontrados. Anopheles (Kerteszia) cruzii é considerado importante mosquito transmissor nessa região, tendo como criadouros águas retidas em bromélias ${ }^{513}$.

A Mata Atlântica caracteriza-se pela diversidade paisagística e pela existência de áreas de grande beleza, que a tornam atrativa para indústria do turismo. Atualmente, restam cerca de 7,3\% de sua cobertura florestal original, porém na região do litoral norte do Estado de São Paulo ocupa aproximadamente 85\% do seu território ${ }^{10}$.

A região constitui pólo de atração turística, cujos municípios totalizam 270 mil habitantes fixos e uma população flutuante estimada em 1.450 .000 pessoas por ano, principalmente nos meses de verão. Esta vulnerabilidade aliada à presença do mosquito transmissor torna esta área de risco à ocorrência de novos casos.

O presente estudo teve como objetivo conhecer a epidemiologia local da malária autóctone por meio da sua distribuição espacial e temporal na região do litoral norte paulista, entre 1985 e 2006, segundo atributos pessoais, densidade parasitária, sintomas e atividade por ocasião de infecção.

\section{MATERIAL E MÉTODOS}

O litoral norte do Estado de São Paulo, compreende quatro municípios: Ubatuba, Caraguatatuba, São Sebastião e Ilhabela; localizadas respectivamente nas coordenadas: latitude sul $23^{\circ} 26^{\prime} 13^{\prime \prime}$ e longitude oeste $45^{\circ} 04^{\prime} 08^{\prime \prime}$, latitude sul $23^{\circ} 29^{\prime} 28^{\prime \prime}$ e longitude oeste $45^{\circ} 25^{\prime} 20^{\prime \prime}$, latitude sul $23^{\circ} 21^{\prime} 20^{\prime \prime}$ e longitude oeste $45^{\circ} 21^{\prime} 00^{\prime \prime}$ e; latitude sul $23^{\circ} 46^{\prime} 28^{\prime \prime}$ e longitude oeste $45^{\circ} 21^{\prime} 28^{\prime \prime}$, respectivamente. A região apresenta clima tropical quente e úmido com temperatura mínima de $15^{\circ} \mathrm{C}$ e máxima de $40^{\circ} \mathrm{C}^{10}$.

A população da região concentra-se na área urbana, 96,1\% em 2005, com elevada taxa de crescimento populacional. Apresenta importante variação sazonal, com registro de 269.781 habitantes fixos, e uma população flutuante composta principalmente por segunda residência de turistas ${ }^{10}$.

Foi realizado um estudo descritivo de uma série temporal de casos de malária autóctone cujos dados foram obtidos na ficha de investigação epidemiológica e entomológica da Superintendência de Controle de Endemias (SUCEN) e sistema de Informação de Notificação de Agravos à Saúde (SINAN).

\section{RESULTADOS}

Os municípios de São Sebastião com 49 e Caraguatatuba com 25 apresentaram maior número de casos, com 59\% e 30,1\%, respectivamente. Ubatuba registrou $8(9,6 \%)$ casos , e Ilhabela 1 (1,3\%) (Tabela 1). A frequiência média anual foi de 4 casos, porém sua distribuição em números absolutos, no período de estudo, foi variável. Os maiores registros foram em 1993, 1994 e 2006 com 8, 10 e 8, casos, respectivamente, sem ocorrência nos anos de 1987, 1999 e 2004 (Figura 1). Foram notificados no período 83 casos, 64 (77\%) do sexo masculino e 19 (23\%), feminino (Tabela 1). Os indivíduos mais acometidos encontraramse nas faixas etárias de 20 - 29 (20,4\%), 30 - 39 (26,5\%) e 40 - $49(21,7 \%)$, esses três grupos totalizam 57 (68,6\%) casos (Tabela 2). Dentre os casos registrados 54 (64\%), residiam na região do litoral norte, e 30 (36\%), eram procedentes de outras áreas. Plasmodium vivax foi o único agente etiológico diagnosticado, com densidade parasitária de um parasita a cada dois ou mais campos microscópicos para a maioria $(68,7 \%)$ dos casos (Tabela 3).

Tabela 1 - Freqüência de casos de malária autóctone, Litoral Norte do Estado de São Paulo, segundo município e sexo, período de 1985 e 2006.

\begin{tabular}{lrrrrr}
\hline & \multicolumn{2}{c}{ Sexo } & & \multicolumn{2}{c}{ Total } \\
\cline { 2 - 3 } \cline { 5 - 6 } Casos/Município & masculino & feminino & & $n^{0}$ & $\%$ \\
\hline São Sebastião & 39 & 10 & & 49 & 59,0 \\
Caraguatatuba & 18 & 7 & & 25 & 30,1 \\
Ubatuba & 6 & 2 & & 9 \\
Ilhabela & 1 & 0 & 1 & 9,6 \\
\hline Total & $\mathbf{6 4 ( 7 7 \% )}$ & $\mathbf{1 9 ( 2 3 \% )}$ & $\mathbf{8 3}$ & $\mathbf{1 0 0 , 0}$ \\
\hline
\end{tabular}

Tabela 2 - Freqüência de casos de malária autóctone, Litoral Norte do Estado de São Paulo, segundo sexo e faixa etária, no período de 1985 a 2006.

\begin{tabular}{|c|c|c|c|c|}
\hline \multirow[b]{2}{*}{ Faixa etária } & \multicolumn{2}{|c|}{ Casos $\left(\mathrm{n}^{0}\right)$} & \multicolumn{2}{|c|}{ Total } \\
\hline & masculino & feminino & $\mathrm{n}^{0}$ & $\%$ \\
\hline $0 \mid-9$ & 1 & 2 & 3 & 3,6 \\
\hline $10 \mid-19$ & 4 & 3 & 7 & 8,4 \\
\hline $20 \mid-29$ & 15 & 2 & 17 & 20,4 \\
\hline $30 \mid-39$ & 18 & 4 & 22 & 26,5 \\
\hline $40 \mid-49$ & 14 & 4 & 18 & 21,7 \\
\hline $50 \mid-59$ & 7 & 1 & 8 & 9,6 \\
\hline $60 \mid-69$ & 3 & 0 & 3 & 3,6 \\
\hline $70 \mid-79$ & 2 & 3 & 5 & 6,2 \\
\hline Total & $64(77 \%)$ & $19(23 \%)$ & 83 & 100,0 \\
\hline
\end{tabular}

Tabela 3 - Freqüência de casos de malária autóctone, Litoral Norte do Estado de São Paulo, segundo agente etiológico e densidade parasitária, no período de 1985 a 2006.

\begin{tabular}{lrr}
\hline & \multicolumn{2}{c}{ Casos } \\
\cline { 2 - 3 } Densidade parasitária & $\mathrm{n}^{0}$ & $\%$ \\
\hline Plasmodium vivax $+/ 2$ & 57 & 68,7 \\
Plasmodium vivax + & 15 & 18,0 \\
Plasmodium vivax ++ & 10 & 12,0 \\
Plasmodium vivax +++ & 1 & 1,3 \\
Plasmodium vivax ++++ & - & - \\
\hline Total & $\mathbf{8 3}$ & $\mathbf{1 0 0 , 0}$ \\
\hline
\end{tabular}




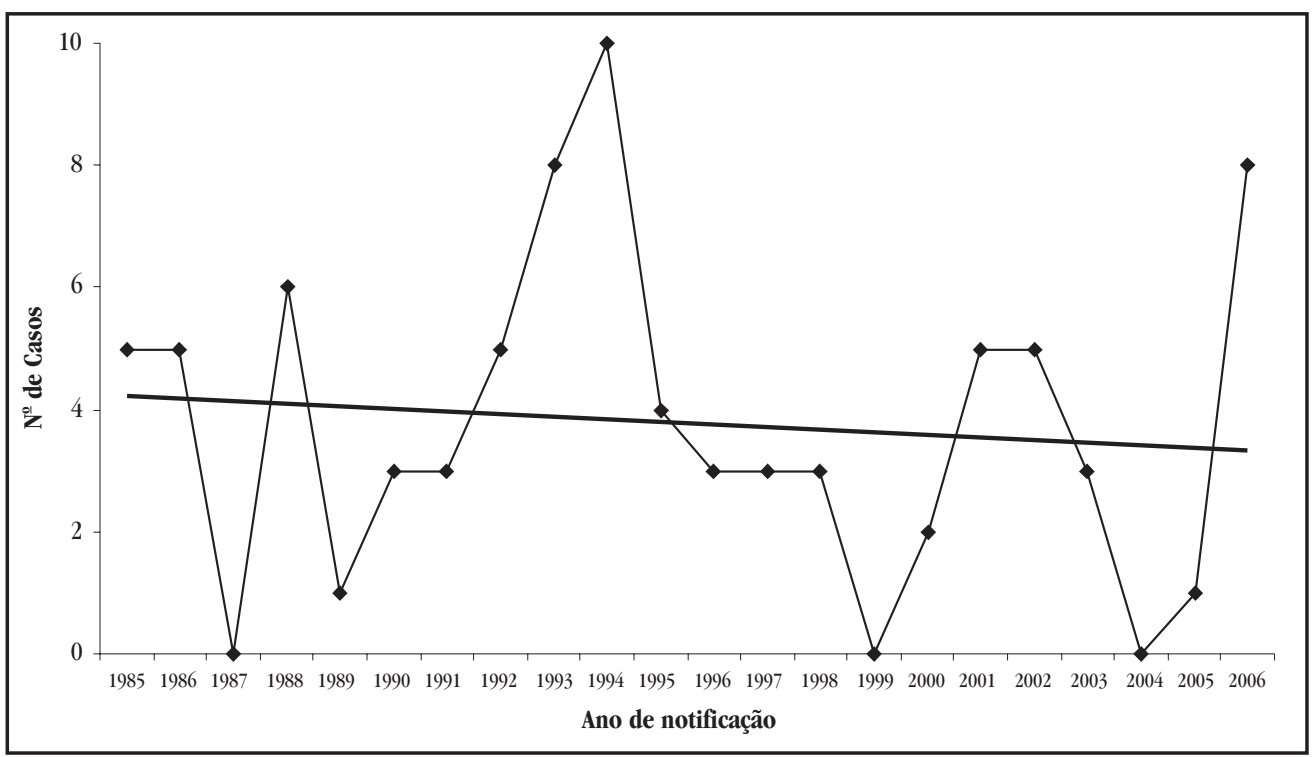

Figura 1 - Número de casos de malária, segundo ano de notificação, Litoral Norte, Estado de São Paulo, no período de 1985 a 2006.

Das atividades exercidas pelos pacientes por ocasião da infecção, o lazer foi registrado em $75 \%$ e o trabalho em $25 \%$ dos casos.

Os principais sintomas foram febre $(90 \%)$, calafrios e cefaléia (74\%), sudorese (53\%), mialgia (50\%) e náuseas (33\%). Foram registrados 4 indivíduos assintomáticos, detectados pela busca ativa de casos.
As capturas entomológicas realizadas nas localidades de transmissão, na ocasião da ocorrência dos casos autóctones totalizaram 226 exemplares imaturos e adultos de diversas espécies, sendo 105 (47\%) anofelinos. Quanto ao sexo, o número de fêmeas superou o de machos, com 95,3\% de frequiência. A espécie Anopheles (Kerteszia) cruzii foi predominante, representando 76\% dos exemplares de anofelinos coletados (Figura 2).

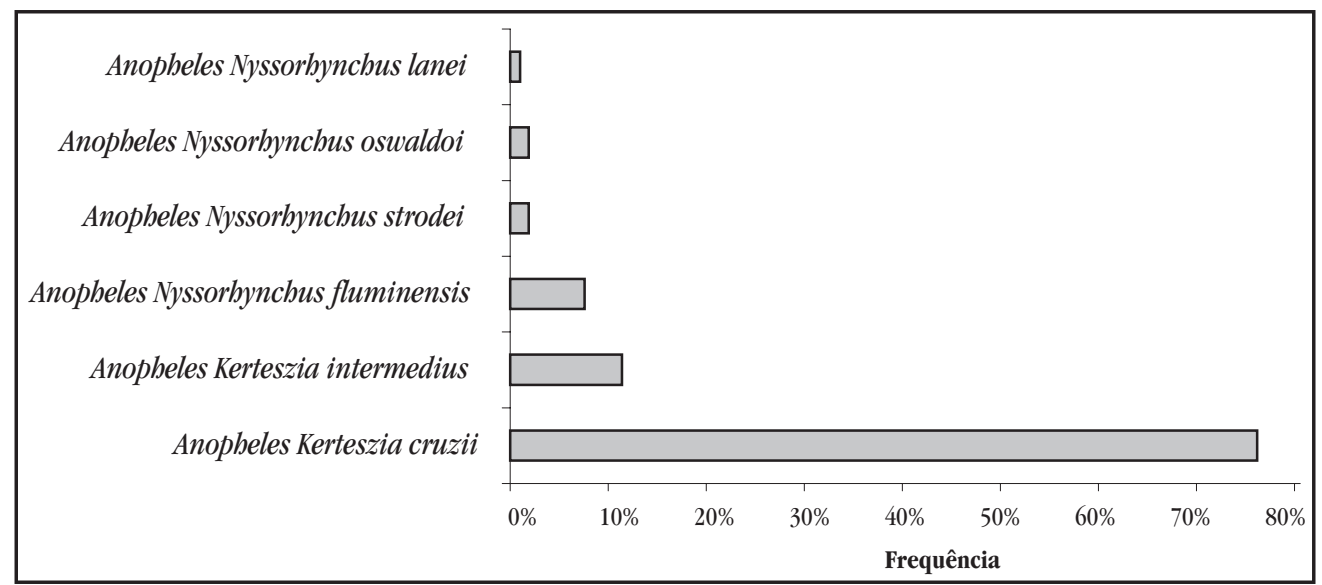

Figura 2 - Frequência de anofelinos capturados em pesquisas entomológicas no Litoral Norte do Estado de São Paulo, no período de 1985 a 2006.

\section{DISCUSSÃo}

A distribuição espacial dos casos de malária no período evidenciou São Sebastião como o município responsável pelo maior número de casos autóctones. Este fato se justifica provavelmente pelo elevado crescimento populacional e incremento do turismo ocorrido nas últimas décadas. A região apresenta elevada (3,8\% aa) taxa de crescimento populacional, duas vezes superior a do Estado de São Paulo ${ }^{11}$.
$\mathrm{Na}$ série temporal, houve ocorrência da doença em praticamente todos os anos do período estudado, com número pequeno de casos. A ausência de casos em alguns anos, não pode caracterizar ausência de transmissão, pois a descentralização do diagnóstico e tratamento da malária nos últimos anos pode ter contribuído para a subnotificação de casos, assim como a existência de indivíduos assintomáticos e a baixa densidade parasitária predominante na região. 0 esperado é que continuem a ocorrerem novos e esporádicos registros de $\operatorname{casos}^{15}$. 
A existência de ambiente ecológico propício ao ecoturismo na região, pode ter favorecido o predomínio da doença no sexo masculino e nas faixas etárias de adultos jovens economicamente ativos, e o lazer como principal atividade por ocasião da infecção. Essas observações são coincidentes com a de vários autores ${ }^{2} 713$.

0 diagnóstico de malária por Plasmodium vivax com baixa parasitemia coincide com o observado em outras regiões cobertas pela Mata Atlântica no Estado de São Paulo ${ }^{16}$.

A presença de assintomáticos detectados na região de estudo deve ser melhor avaliada. A relação entre doença e parasitemia depende da espécie do parasita, virulência da cepa e imunidade do paciente. Estes pacientes, sem diagnóstico e sem tratamento oferecem a possibilidade de manter a circulação do agente etiológico e produzir novos casos. Não está claro por que ocorrem infecções assintomáticas atribuídas a Plasmodium vivax em áreas de transmissão autóctone em São Paulo. Em muitas regiões do Brasil, pode estar associada à exposição cumulativa ao parasita ${ }^{10}$, já na região do Litoral Sul Paulista, é atribuída à circulação de variantes de Plasmodium vivax e de plasmódios símios ${ }^{34}$.

0 ecossistema preservado, ecoturismo, e a presença de Kerteszia são fatores que potencializam o risco de transmissão de malária autóctone na região.

A ocorrência da doença se deu em indivíduos que necessariamente frequientaram ambiente preservado, Mata Atlântica, que encerra a presença de vetores transmissores da doença. Portanto, deve-se valorizar na investigação epidemiológica, os aspectos locais de transmissão.

A detecção de assintomáticos indica a manutenção da circulação do Plasmodium na região e conseqüientemente favorecerá o registro de novos casos. Há, portanto, necessidade de novas estratégias para revelar a presença de portadores de malária, como técnicas mais sensíveis de diagnóstico para detectar indivíduos assintomáticos.

Os dados apontados neste estudo devem constituir aspectos importantes para um sistema de informação de casos, de vetores, de situações ambientais para procedimentos de controle da doença na região.

A melhoria das ações de vigilância e controle da malária no Litoral Norte Paulista deve ser obtida por meio de estratégias que combinem: suspeita clínica e/ou epidemiológica, existência de laboratório de referência e recursos humanos treinados para 0 diagnóstico e tratamentos adequados.

\section{REFERÊNCIAS}

1. Alves MJCP, Mayo RC, Donalísio MR. História, Epidemiologia e Controle da Malária na região de Campinas, Estado de São Paulo, Brasil, 1980 a 2000. Revista da Sociedade Brasileira Medicina Tropical 37:41-45, 2004.

2. Barroso WJ. A Malária de Mata Atlântica no Brasil. Informe Farmacêutico 15: 1-3, 2003.

3. Branquinho MS, Marrelli MT, Curado I, Natal D, Barata JMS, Tubaki R, Carréri-Bruno GC, Menezes RT, Kloetzel JK. Infecção do Anopheles (Kerteszia) cruzii por Plasmodium vivax e Plasmodium vivax variante VK 247 nos municípios de São Vicente e Juquitiba, São Paulo. Revista Panamericana Salud Pública 2:189-193, 1997.

4. Curado I, Malafronte RS, Duarte AMRC, Kirchgatter K, ranquinho MS, EAB Galati. Malaria epidemiology in low-endemicity areas of the Atlantic Forest in the Vale do Ribeira, São Paulo, Brazil. Acta Tropica 100:54-62, 2006.

5. Forattini OP. Culicidologia Médica: Identificação, Biologia e Epidemiologia, $1^{\text {a }}$ edição, Editora Edusp, São Paulo 383-397, 2002.

6. Junqueira FM, Tangerino JC, Silva MV, Rocha MCP, Nogueira SLR, Pereira BF. Surto de Malária assintomática em área de baixa endemicidade no Estado de São Paulo. In: Resumos do XLIII Congresso Sociedade Brasileira de Medicina Tropical, II Encontro de Medicina Tropical dos Países de Língua Portuguesa, I Encontro da Sociedade Brasileira de Medicina de Viagens. Campos do Jordão, p. 1432007.

7. Machado RLD, D'Almeida Couto AAR, Cavasini CE, Calvosa VSP. Malária em região extra-Amazônica: situação no Estado de Santa Catarina. Revista da Sociedade Brasileira Medicina Tropical 36:581-586, 2003.

8. Ministério da Saúde. Secretaria de Vigilância em Saúde. Guia de Vigilância Epidemiológica. 6a edição, Brasília, p.444-466, 2006.

9. Ministério da Saúde. Secretaria de Vigilância em Saúde. Situação Epidemiológica da Malária no Brasil. 2007.

10. Secretaria de Estado do Meio Ambiente - SEMA. Coordenadoria de Planejamento Ambiental Estratégico e Educação Ambiental. Decreto SMA n49.215, de 7 de dezembro de 2004. Zoneamento Ecológico-Econômico do Litoral Norte. SMA/CPLEA, São Paulo, 2005.

11. Silva RSU, Santos ECO, Loureiro ECB, Jesus IM, Souza V, Brabo EES. Malária assintomática - uma das causas da perpetuação da transmissão da malária. In: Anais da IV Reunião Nacional dos Pesquisadores em malária, Manaus, p. 31, 1993.

12. Sistema Estadual de Análise de Dados. Sistemas de informações dos municípios paulistas. Disponível na URL: http://www.seade.gov.br/produtos/imp/imp.php. Acesso em 22 junho 2007, 2006.

13. Superintendência de Controle de Endemias - Secretaria da Saúde do estado de São Paulo. Manual de Malária: Situação atual, diagnóstico, tratamento e controle. Programa de Controle da Malária no Estado de São Paulo, São Paulo, 1988.

14. Ueno HM, Forattini OP, Kakitani I. Distribuição vertical e sazonal de Anopheles (Kerteszia) em Ilha Comprida, SP. Revista de Saúde Pública 41:269-275, 2007.

15. Wanderley DMV, Silva RA, Andrade JCR. Aspectos epidemiológicos da malária no Estado de São Paulo, Brasil, 1983 a 1992. Revista de Saúde Pública 28:192-197, 1994

16. Wanderley DMV, Ciaravolo RMC, Barbosa G L, Spínola R, Leite RM. Malária no Estado de São Paulo: Aspectos da Vigilância Epidemiológica. Boletim Epidemiológico Paulista. 2-7 agosto, 2006. 